\title{
A REMARK ON THE ESSENTIAL SPECTRA OF QUASI- SIMILAR DOMINANT CONTRACTIONS
}

\author{
by B. P. DUGGAL
}

(Received 18 November, 1987)

Introduction. We consider operators, i.e. bounded linear transformations, on an infinite dimensional separable complex Hilbert space $H$ into itself. The operator $A$ is said to be dominant if for each complex number $\lambda$ there exists a number $M_{\lambda}(\geqslant 1)$ such that $\left\|(A-\lambda)^{*} x\right\| \leqslant M_{\lambda}\|(A-\lambda) x\|$ for each $x \in H$. If there exists a number $M \geqslant M_{\lambda}$ for all $\lambda$, then the dominant operator $A$ is said to be $M$-hyponormal. The class of dominant (and $M$-hyponormal) operators was introduced by J. G. Stampfli during the seventies, and has since been considered in a number of papers, amongst then [7], [11]. It is clear that a 1-hyponormal is hyponormal. The operator $A$ is said to be quasi-normal if $A$ commutes with $A^{*} A$, and we say that $A$ is subnormal if $A$ has a normal extension. It is known that the classes consisting of these operators satisfy the following strict inclusion relation:

$$
\text { quasi-normal } \subset \text { subnormal } \subset \text { hyponormal } \subset M \text {-hyponormal } \subset \text { dominant }
$$

We say that the operator $X$ is a quasi-affinity if both $X$ and $X^{*}$ have dense range. Given operators $A, B$ and $X$, let $C(A, B) X=A X-X B$. The operators $A$ and $B$ are said to be quasi-similar, denoted $A \sim B$, if there exist quasi-affinities $X$ and $Y$ such that $C(A, B) X=C(B, A) Y=0$, and we say that $A \stackrel{d}{\sim} B$ if there exist operators $X$ and $Y$ with dense range such that $C(A, B) X=C(B, A) Y=0$. Given the operator $A$, let $\sigma(A)$ and $\sigma_{e}(A)$ denote, respectively, the spectrum and the essential spectrum of $A$.

The problem of the equality of the spectra, and the essential spectra, of quasi-similar operators has been considered by a number of authors in the recent past (see [1], [2], [5], [8], [9], [10], [11]). Recall that each operator $A$ has a direct sum decomposition $A=A_{1} \oplus A_{2}$, where $A_{1}$ is normal and $A_{2}$ is pure. (The operator $A_{2}$ is said to be pure if there exists no non-trivial reducing subspace $M$ of $A_{2}$ such that $A_{2} \mid M$, the restriction of $A_{2}$ to $M$, is normal. It is to be noted here that either component in the direct sum $A=A_{1} \oplus A_{2}$ may be absent.) Given quasi-similar dominant operators $A$ and $B$ such that $A=A_{1} \oplus A_{2}$ and $B=B_{1} \oplus B_{2}$, it is fairly easily seen that $A_{1}$ and $B_{1}$ are unitarily equivalent, and that $A_{2} \stackrel{d}{\sim} B_{2}$ [11, Theorem 1.1]. The problem of determining whether $\sigma_{e}(A)=\sigma_{e}(B)$ (or, $\sigma(A)=\sigma(B)$ ) then reduces to that of determining whether $\sigma_{e}\left(A_{2}\right)=$ $\sigma_{e}\left(B_{2}\right)$ (respectively, $\sigma\left(A_{2}\right)=\sigma\left(B_{2}\right)$ ). It is to be noted here that the operators (with dense ranges) intertwining $A_{2}$ and $B_{2}$ need not be quasi-affinities, even in the case in which $A_{2}$ and $B_{2}$ are quasi-normal [9]. However, the fact that these intertwining operators lack injectivity seems to play but a minor role. It is known that if $A_{2}$ and $B_{2}$ are quasi-normal, then $\sigma_{e}\left(A_{2}\right)=\sigma_{e}\left(B_{2}\right)$ [9] (and that if $A_{2}$ and $B_{2}$ are hyponormal, then $\sigma\left(A_{2}\right)=\sigma\left(B_{2}\right)$ [1], [8]): the problem, however, remains unsolved for subnormal $A_{2}$ and $B_{2}$. Assuming additional hypotheses, such as that one of the quasi-affinities intertwining $A$ and $B$ is compact, L. R. Williams [10] has shown that $\sigma_{e}(A)=\sigma_{e}(B)$ if $A$ and $B$ are hyponormal.

Glasgow Math. J. 31 (1989) 165-168. 
(That a similar result holds for $k$-quasihyponormal $A$ and $B$ has been shown by B. C. Gupta [5].) The result for hyponormal $A$ and $B$ extends to dominant $A$ and $B$ if $A$ and $B$ satisfy Dunford's Condition $C$ [11, Theorem 2.4].

In this note we consider contractions $A$ and $B$, and show that if $A$ is injective, then the hypothesis that the dominant $A$ and $B$ satisfy Dunford's Condition $C$ can be dispensed with in [11, Theorem 2.4]. Indeed we show more. It is known that the c.n.u. (= completely non-unitary) part of a dominant contraction is of the class $C_{.0}$ of contractions [4]. We show that if $A$ and $B$ are quasi-similar contractions with $C_{.0}$ c.n.u. parts, if one of the intertwining quasi-affinites is compact, and if $A$ is injective, then $A$ and $B$ are hyponormal, and so $\sigma_{e}(A)=\sigma_{e}(B)$.

Results. A contraction $A$ is said to be c.n.u. if there exists no non-trivial reducing subspace $M$ of $A$ such that $A \mid M$ is unitary. Recall that every contraction $A$ has a direct sum decomposition $A=A_{1} \oplus A_{2}$, where $A_{1}$ is unitary and $A_{2}$ is c.n.u. (and where either of the components may be absent). We say that the contraction $A$ belongs to the class $C_{0}$ (class $C_{.1}$ ) of contractions if $\left\|A^{* n} x\right\| \rightarrow 0$ as $n \rightarrow \infty$ (respectively, $0<\inf _{n}\left\|A^{* n} x\right\|$ ) for each non-trivial $x \in H$. The classes $C_{0}$ and $C_{1}$ are defined by considering $A^{*}$ instead of $A$, and we say that $A \in C_{\alpha \beta}$, where $\alpha, \beta=0,1$, if $A \in C_{\alpha .} \cap C_{\beta}$. In the following we shall denote the point spectrum of $A$ by $\sigma_{p}(A)$, the closure of the range of $A$ by $\overline{\operatorname{ran}} A$, and the orthogonal complement of the kernel of $A$ by $\operatorname{ker}^{\perp} A$. We now prove our result for the case in which $A$ and $B$ are dominant: the case of general contractions with $C_{.0}$ c.n.u. parts will be seen to follow from the proof of this case.

Theorem 1. Let $A$ and $B$ be dominant contractions such that $0 \notin \sigma_{p}(A)$ and $A \sim B$. If one of the quasi-affinities intertwining $A$ and $B$ is compact, then $\sigma_{e}(A)=\sigma_{e}(B)$.

Proof. Let $X$ and $Y$ be the quasi-affinities, with $Y$ compact, such that $C(A, B) X=$ $C(B, A) Y=0$. We show that $A$ and $B$ are hyponormal, and this is achieved by showing that the c.n.u. parts of $A$ and $B$ are hyponormal. Thus, decompose $A$ and $B$ into their unitary and c.n.u. parts by $A=A_{1} \oplus A_{0}$ and $B=B_{1} \oplus B_{0}$. Since $A$ and $B$ are dominant, $A_{0}$ and $B_{0} \in C_{.0}[4]$, and so have triangulations

$$
\left[\begin{array}{cc}
A_{2} & { }^{*} \\
0 & A_{3}
\end{array}\right] \text { and }\left[\begin{array}{cc}
B_{2} & \\
0 & B_{3}
\end{array}\right] \text { of the type }\left[\begin{array}{cc}
C_{00} & { }^{*} \\
0 & C_{10}
\end{array}\right]
$$

[6, p. 75]. Let $X$ and $Y$ have the corresponding matrix representations

$$
X=\left[X_{i j}\right]_{i, j=1}^{3} \text { and } Y=\left[Y_{i j}\right]_{i, j=1}^{3} \text {. }
$$

Then, since $A_{1}$ and $B_{1} \in C_{11}, A_{2}$ and $B_{2} \in C_{00}$, and $A_{3}$ and $B_{3} \in C_{10}$, it follows from the equations $0=C\left(A_{1}, B_{2}\right) X_{12}=C\left(B_{1}, A_{2}\right) Y_{12}=C\left(A_{2}, B_{1}\right) X_{21}=C\left(B_{2}, A_{1}\right) Y_{21}=$ $C\left(A_{3}, B_{1}\right) X_{31}=C\left(B_{3}, A_{1}\right)_{31}=C\left(A_{3}, B_{2}\right) X_{32}=C\left(B_{3}, A_{2}\right) Y_{32}$ that $X_{12}=Y_{12}=X_{21}=$ $Y_{21}=X_{31}=Y_{31}=X_{32}=Y_{32}=0$. (Sample argument: since $A_{1}$ is unitary and $B_{3} \in C_{10}$, $\left\|Y_{31}^{*} x\right\|=\left\|A_{1}^{* n} Y_{31}^{*} x\right\|=\left\|Y_{31}^{*} B_{3}^{* n} x\right\| \leqslant\left\|Y_{31}^{*}\right\|\left\|B_{3}^{* n} x\right\| \rightarrow 0$ as $n \rightarrow \infty$.) Consequently, $X$ and $Y$ 
have the representations

$$
\left[\begin{array}{ccc}
X_{11} & 0 & X_{13} \\
0 & X_{22} & X_{23} \\
0 & 0 & X_{33}
\end{array}\right] \text { and }\left[\begin{array}{ccc}
Y_{11} & 0 & Y_{13} \\
0 & Y_{22} & Y_{23} \\
0 & 0 & Y_{33}
\end{array}\right] \text {, }
$$

where $X_{33}$ and $Y_{33}$ have dense range (with $Y_{33}$ compact). We now consider the equations $C\left(A_{3}, B_{3}\right) X_{33}=C\left(B_{3}, A_{3}\right) Y_{33}=0$, and show that $A_{3}$ and $B_{3}$ are in fact non-existent.

Set $X_{33} Y_{33}=S_{1}$; then $S_{1}$ is a compact operator with dense range such that $C\left(A_{3}, A_{3}\right) S_{1}=0$. Since $H$ is separable and $A_{3} \in C_{10}$, there exists an isometry $V$ and a quasi-affinity $S_{0}$ such that $C\left(V, A_{3}\right) S_{0}=0$ [6, Proposition II.3.5]. Set $S_{0} S_{1}=S$; then $S$ is a compact operator with dense range such that $C\left(V, A_{3}\right) S=0$, and hence that $A_{3}^{*} S^{*} S A_{3}=$ $S^{*} S$. This implies that $\overline{\operatorname{ran}} S^{*} S=\operatorname{ker}^{\perp} S$ reduces $A_{3}$ and $A_{3} / \operatorname{ker}^{\perp} S$ is unitary (see [3, Theorem 8 and Corollary 6.5]). Since $A_{3}$ is c.n.u., we conclude that $A_{3}$ is non-existent. By symmetry, $B_{3}$ is also non-existent. Consequently, $X_{13}=Y_{13}=0$.

The preceding argument shows that $C\left(A_{0}, B_{0}\right) X_{0}=C\left(B_{0}, A_{0}\right) Y_{0}=0$, where

$$
X_{0}=\left[\begin{array}{cc}
X_{22} & X_{23} \\
0 & X_{33}
\end{array}\right] \text { and } Y_{0}=\left[\begin{array}{cc}
Y_{22} & Y_{23} \\
0 & Y_{33}
\end{array}\right]
$$

are quasi-affinities (with $Y_{0}$ compact). Set $X_{0} Y_{0}=T$; then $\left(: H^{\prime} \rightarrow H^{\prime}\right.$, say) is a compact quasi-affinity such that $C\left(A_{0}, A_{0}\right) T=0$. Clearly, $0 \notin \sigma_{p}\left(A_{0}\right)$ and $0 \notin \sigma_{p}\left(B_{0}\right)$. Since $A_{0} \in C_{.0}$, the sequence $\left\{A_{0}^{n} T T^{*} A_{0}^{* n}\right\}$ converges strongly to 0 , and so, since $A_{0} T T^{*} A_{0}^{*}=$ $T A_{0} A_{0}^{*} T^{*} \leqslant T T^{*}, T T^{*}$ is a 'pure solution' (in the sense of [3, p. 24]) of $A_{0} T T^{*} A_{0}^{*} \leqslant T T^{*}$. Hence there exists a unilateral shift $U$ (on a Hilbert space $\mathscr{H}$ ) and an operator $C$ (on $\left.H^{\prime} \rightarrow \mathscr{H}\right)$ such that $C A_{0}^{*}=U^{*} C$ and $T T^{*}=C^{*} C[3$, Theorem 5]. Clearly, there exists an isometry $V: \mathscr{H} \rightarrow H^{\prime}$ (indeed, $V$ is a unitary on $\operatorname{ran} C \rightarrow H^{\prime}$ ) such that $T^{*}=V C$, and so that

$$
A_{0}^{*} T^{*}=T^{*} A_{0}^{*}=V C A_{0}^{*}=V U^{*} V^{*} V C=V U^{*} V^{*} T^{*} .
$$

Since $T^{*}$ is a quasi-affinity, this implies that $A_{0}^{*}=V U^{*} V^{*}$, and hence that $A_{0}$ is hyponormal. Consequently, $A$ is hyponormal. By symmetry, $B$ is also hyponormal.

It now follows that $A$ and $B$ are quasi-similar hyponormal contractions. Hence $\sigma_{e}(A)=\sigma_{e}(B)$, by [10], as was to be proved.

Remarks. (1). The proof of the theorem shows that $A_{0} \sim B_{0}$ : this does not in any way imply that the pure parts of $A$ and $B$ are quasi-similar. As mentioned earlier, it can be seen using a routine argument that the pure parts of $A$ and $B$, say $A_{4}$ and $B_{4}$, satisfy $A_{4} \stackrel{d}{\sim} B_{4}$.

(2). The argument in the proof above leading to the conclusion that $A_{0}$ is hyponormal fails for a general quasi-affinity. This is easily seen upon letting $\left\{e_{n}\right\},-\infty<$ $n<\infty$, be an orthonormal basis of $H$, (the dominant contraction) $A_{0}$ be the bilateral shift $A_{0} e_{n}=2^{-|n|} e_{n+1}$ and $T$ be the quasi-affinity defined by $T e_{n}=2^{-1} e_{n}$.

(3). The hypothesis that $A$ is injective seems to be required in Theorem 1 (just as it seems that some such hypothesis is required in [3, Theorem 5]) in the sense that the 
unilateral shift $U$ may otherwise fail to exist. For suppose that $A_{0}$ is not injective and that $A_{0}=V U V^{*}$. Then there exists a non-trivial $x$ such that $A_{0} x=0$, and hence that $V^{*} x=0$. Since $T=C^{*} V^{*}$, we have that $T x=0$, i.e., $T$ is not injective.

(4). A scrutiny of the proof above shows that the fact that $A$ and $B$ are dominant plays a part in the proof only through the fact that they have $C_{.0}$ c.n.u. parts. Hence Theorem 1 generalises to the following result.

THEOREM 2. Let $A$ and $B$ be contractions with $C_{.0}$ c.n.u. parts such that $0 \notin \sigma_{p}(A)$ and $A \sim B$. If one of the quasi-affinities intertwining $A$ and $B$ is compact, then $\sigma_{e}(A)=\sigma_{e}(B)$.

\section{REFERENCES}

1. S. Clary, Equality of spectra of quasi-similar hyponormal operators, Proc. Amer. Math. Soc. $\mathbf{5 3}$ (1975), 88-90.

2. J. B. Conway, Subnormal operators (Pitman, 1981).

3. R. G. Douglas, On the operator equation $S^{*} X T=X$ and related topics, Acta Sci. Math. (Szeged) 30 (1969), 19-32.

4. B. P. Duggal, Intertwining contractions: operator equation $A X B=X$ and $A X=X B$, Proc. Int. Conf. on 'Invariant subspaces and allied topics', University of Delhi, Dec. 1986 (to appear). 49-54.

5. B. C. Gupta, Quasi-similarity and $k$-quasihyponormal operators, Math. Today 3 (1985),

6. B. Sz.-Nagy and C. Foias, Harmonic analysis of operators on Hilbert space, (NorthHolland, 1970).

7. J. G. Stampfli and B. L. Wadhwa, An asymmetric Putnam-Fuglede theorem for dominant operators, Indiana Univ. Math. J. 25 (1976), 359-365. 109-119.

8. J. G. Stampfli, Quasi-similarity of operators, Proc. Roy. Irish Acad. Sect. A 81 (1981),

9. L. R. Williams, Equality of essential spectra of quasi-similar quasinormal operators, $J$. Operator Theory 3 (1980), 57-69.

10. L. R. Williams, Equality of essential spectra of certain quasi-similar semi-normal operators, Proc. Amer. Math. Soc. 78 (1980), 203-209.

11. L. R. Williams, Quasi-similarity and hyponormal operators, J. Operator Theory 5 (1981), 127-139.

School of Mathematical Sciences

UNIVERSITY OF KHARTOUM

P.O. Box 321

KHARTOUM

SUDAN 